\title{
Inhibitory Effects of Brassicaceae Cover Crop on Ambrosia artemisiifolia Germination and Early Growth
}

\author{
Maja Šćepanović ${ }^{1}\left(\right.$, , Marija Sarić-Krsmanović ${ }^{2}$, Valentina Šsštarčić ${ }^{1, *}$, Ema Brijačak $^{1}{ }^{1}$, Josip Lakić ${ }^{1}$, \\ Bojana Špirović Trifunović ${ }^{3}$, Jelena Gajić Umiljendić ${ }^{2}$ and Ljiljana Radivojević ${ }^{2}$ \\ 1 Faculty of Agriculture, University of Zagreb, Svetosimunska cesta 25, 10000 Zagreb, Croatia; \\ mscepanovic@agr.hr (M.Š.); ebrijacak@agr.hr (E.B.); jlakic@agr.hr (J.L.) \\ 2 Institute for Pesticide and Environmental Protection, Banatska 31 b, 11080 Belgrade, Serbia; \\ marijasaric.msaric@gmail.com (M.S.-K.); pecikos@gmail.com (J.G.U.); lilijana.radivojevic@pesting.org.rs (L.R.) \\ 3 Faculty of Agriculture, University of Belgrade, 6 Nemanjina Street, 11000 Belgrade, Serbia; \\ spirovic@agrif.bg.ac.rs \\ * Correspondence: vsostarcic@agr.hr; Tel.: +385-1-239-4086
}

\section{check for}

updates

Citation: Šćepanović, M.; Sarić-Krsmanović, M.; Šoštarčić, V.; Brijačak, E.; Lakić, J.; Špirović Trifunović, B.; Gajić Umiljendić, J.; Radivojević, L. Inhibitory Effects of Brassicaceae Cover Crop on Ambrosia artemisiifolia Germination and Early Growth. Plants 2021, 10, 794. https:// doi.org/10.3390/plants10040794

Academic Editor: Nicholas

Emmanuel Korres

Received: 10 March 2021

Accepted: 15 April 2021

Published: 17 April 2021

Publisher's Note: MDPI stays neutral with regard to jurisdictional claims in published maps and institutional affiliations.

Copyright: (c) 2021 by the authors. Licensee MDPI, Basel, Switzerland. This article is an open access article distributed under the terms and conditions of the Creative Commons Attribution (CC BY) license (https:// creativecommons.org/licenses/by/ $4.0 /)$.

\begin{abstract}
Several cover crops (CCs) exert allelopathic effects that suppress weed growth. The aim of the present study was to evaluate the effects of aqueous extracts containing different concentrations $[0,0.5,1,2.5,5,7.5$ and $10 \%(w / v)]$ of Brassicaceae CCs (Sinapis alba, Raphanus sativus, Camellina sativa) and of the CCs Fagopyrum esculentum and Guizotia abyssinica on germination and early growth of Ambrosia artemisiifolia $\mathrm{L}$. The allelopathic effects were species and concentration-dependent. C. sativa, for example, caused the greatest potential to inhibit germination, shoot, radicle length and fresh seedling weight, whereas $S$. alba and $R$. sativus inhibited germination and early growth of A. artemisiifolia only at concentrations $\geq 7.5 \%$. In contrast, no inhibition was observed when aqueous extracts of $F$. escultneum and G. abyssinica were added at any of tested concentration. Liquid chromatography-tandem mass spectrometry detected 15 phenolic compounds in Brassicaceae CCs with the highest content $(\mu \mathrm{g} / \mathrm{g})$ of vanillin (48.8), chlorogenic acid (1057), vanilic acid (79), caffeic acid (102.5) and syringic acid (27.3) in C. sativa. Our results suggest that $C$. sativa is the most allelopathic CCs and that the fruits of $C$. sativa are the plant organs richest in allelochemicals.
\end{abstract}

Keywords: common ragweed; allelopathic potential; Camelina sativa; Raphanus sativus; Sinapis alba; integrated weed control; aqueous extracts; phenolic compounds

\section{Introduction}

Ambrosia artemisiifolia $\mathrm{L}$. is an invasive alien species that originated in North America and is considered one of the most harmful plant species in Europe [1]. This monoecious species produces up to eight million allergen pollen grains [2]. Although it can grow in a wide range of habitat types and climates [1], the highest annual pollen amounts are measured in Pannonian Plain, i.e., European countries with a continental climate and mid-latitude [3]. In fact, Croatia is among the top three European countries in terms of density of pollen and seeds per area produced by uncontrolled $A$. artemisiifolia plants [4]. In the continental part of Croatia, this species is the most abundant weed species in nearly all summer crops [5] and is usually controlled with herbicides [6]. However, the emergence of herbicide-resistant biotypes worldwide [7], the decreasing number of herbicides available on the market [8], and the generally negative ecotoxicological properties of pesticides, combined with social demands towards pesticide-free agriculture, require other approaches to eliminate weeds from the cultivated landscape. Specifically, A. artemisiifolia has developed resistance to several herbicide groups [7] and recently, the poorer efficacy of some postemergence herbicides in soybean has also been reported by Croatian farmers.

The use of cover crops (CCs) is a well-accepted practice in conversation agricultural systems or organic farming to reduce erosion, increase soil biological activity and prevent 
nutrient leaching [9-13]. It is also a useful tool for suppressing weed growth [14,15] in those systems since the presence of cover crops is often negatively correlated to weed biomass [9]. Mostly, the effect of cover crops on weed establishment and development can be considered in two ways: their competitive ability and their allelopathic potential by releasing various allelochemicals [10,15-17]. The idea of competing vegetation as a sustainable control method for A. artemisiifolia has already been demonstrated [18] and further elaborated by a comprehensive field study at 39 sites in Europe, where positive effects of neighboring vegetation in reducing this weed species were demonstrated [4]. However, the possibility of CCs to suppress A. artemisiifolia through allelopathy is still unclear.

Several crops, especially from the Poaceae, Asteraceae and Brassicaceae families, demonstrate strong weed suppression ability by exuding allelochemical compounds from the living plant parts or decomposing residues [9]. Brassicaceae family plants from the Brassica, Camelina, Raphanus and Sinapis tribes are frequently cited as allelopathic [19,20]. A promising way to use allelopathy in weed control is using water extracts of allelopathic plants as herbicides [21]. Indeed, high-weed germination inhibition caused by the residues of those species has already been proved in a greenhouse experiment [22] and usually correlated with the presence of different phenols, the most active allelochemicals, in plant tissue $[10,20,22,23]$. Most allelochemicals, including phenols, are water-soluble, so aqueous extraction is the easiest way to isolate them [24]. Therefore, the aim of the present study is to examine whether aqueous extracts from CCs can indeed inhibit germination and early growth of A. artemisiifolia. In addition, to find out the optimal time for the incorporation of CCs, we used one of the most prominent CCs in the study and evaluated which of the plant organs had the highest allelopathic potential. To address this question, the weed was exposed to different concentrations of aqueous extracts of CCs, and the effects on $A$. artemisiifolia germination, germination rate and early growth were examined. To determine whether Brassicaceae CCs in combination with plant species from other families have a higher allelopathic effect as previously suggested [10,14], aqueous extracts of Brassicaceae CCs and CCs from other families were used in the experiment. Since there is no information in the literature on the sensitivity of A. artemisiifolia to aqueous extracts of different CCs, several concentrations of each CC were used $[20,25]$ to determine the CC with the highest allelopathic potential. Finally, liquid chromatography-tandem mass spectrometry was used to detect phenolic compounds in CCs and correlate them with their inhibitory potential against $A$. artemisiifoilia.

\section{Results}

\subsection{Allelopathic Effects of Aqueous Extracts on Germination and Early Growth of A. artemisiifolia}

The suppression of $A$. artemisiifolia germination depended on the type of CC used and the concentration of $\mathrm{CC}$ in the aqueous extract. Germination was inhibited only by aqueous extracts of Brassicaceae CCs, C. sativa or S. alba, or when all CCs were mixed together ((CCMIX), Table 1$)$. At all concentrations $\geq 2.5 \%$, the aqueous extracts of $C$. sativa significantly inhibited germination to extents ranging from $28.3 \%$ to $42.5 \%$. The extracts of S. alba and CCMIX at a concentration of at least $10 \%$ significantly inhibited germination by $25.6 \%$ and $39.1 \%$, respectively.

Similarly, A. artemisiifolia shoot length, radicle length and seedling fresh weight were reduced to the greatest extent by $10 \%$ extracts of the Brassicaceae C. sativa, R. sativus and $S$. alba or of all CCs mixed together (Table 2). The data indicate that $C$. sativa aqueous extracts exerted stronger allelopathic effects than the other extracts at nearly all concentrations. For example, at all concentrations $(0.5 \%-10 \%)$, the aqueous extract of $C$. sativa resulted in significantly shorter shoots than under control conditions, and the shortening varied from $18.9 \%$ to $54.2 \%$ with increasing concentration. Similarly, significantly shorter radicles ( $41.9 \%-81.8 \%$ reduction) and smaller seedlings ( $23.9 \%-51.9 \%$ reduction) of $A$. artemisiifolia were observed under $C$. sativa extracts but at a concentration $\leq 5 \%$. When all CCs were mixed together (CCMIX), only the radicle of $A$. artemisiifiolia was reduced at concentration $\geq 5 \%$, but other parameters (shoot and seedling fresh weight) were not affected by any 
concentration. Moreover, only the highest concentration (10\%) of S. alba and R. sativa extract was required to inhibit all three measured early growth parameters of $A$. artemisiifolia. Finally, the concentrations of F. esculentum or G. abssynica did not reduce any of the early growth parameters of A. artemisiifolia.

Table 1. Allelopathic effect of different concentrations of aqueous extracts of cover crops on A. artemisiifolia germination.

\begin{tabular}{|c|c|c|c|c|c|c|}
\hline \multirow{2}{*}{$\begin{array}{c}\text { Extract } \\
\text { Concentra- } \\
\text { tion } \\
(w / v)\end{array}$} & \multicolumn{6}{|c|}{ Cover Crop (s) } \\
\hline & $\begin{array}{l}\text { All Crops Mixed } \\
\text { (CCMIX) }\end{array}$ & C. sativa & F. esculentum & G. abyssinica & R. sativus & S. alba \\
\hline Control & $75.00 \pm 2.35^{\mathrm{A}-\mathrm{F} \mathrm{ab}}$ & & & & & \\
\hline 0.5 & $68.13 \pm 2.91^{\mathrm{C}-\mathrm{F} \mathrm{ab}}$ & $67.50 \pm 2.08^{\mathrm{C}-\mathrm{F} a b}$ & $76.88 \pm 2.81^{\mathrm{A}-\mathrm{C} \mathrm{a}}$ & $79.38 \pm 1.25^{\mathrm{A}-\mathrm{Ca}}$ & $77.50 \pm 2.54^{\mathrm{A}-\mathrm{C} \mathrm{ab}}$ & $83.33 \pm 3.12^{\mathrm{A} \mathrm{a}}$ \\
\hline 1.0 & $76.88 \pm 2.75^{\mathrm{D}-\mathrm{Ga}}$ & $56.88 \pm 1.66^{\mathrm{E}-\mathrm{H} \mathrm{bc}}$ & $78.13 \pm 2.27 \mathrm{~A}-\mathrm{C} \mathrm{a}$ & $76.25 \pm 2.99 \mathrm{~A}-\mathrm{C} \mathrm{a}$ & $78.13 \pm 2.73^{\mathrm{A}-\mathrm{C} \mathrm{a}}$ & $81.67 \pm 2.24 \mathrm{AB} \mathrm{a}$ \\
\hline 2.5 & $63.75 \pm 1.92^{\mathrm{D}-\mathrm{G} \mathrm{ab}}$ & $53.75 \pm 1.51^{\mathrm{G}-\mathrm{I} b c}$ & $76.88 \pm 2.54^{\mathrm{A}-\mathrm{C} \mathrm{a}}$ & $74.38 \pm 2.39 \mathrm{~A}-\mathrm{D}$ a & $72.50 \pm 2.40^{\mathrm{A}-\mathrm{D} a b}$ & $72.50 \pm 2.40^{\mathrm{A}-\mathrm{D} a b}$ \\
\hline 5.0 & $55.00 \pm 1.42^{\mathrm{G}-\mathrm{I} b c}$ & $51.25 \pm 1.44 \mathrm{HI} \mathrm{c}$ & $73.75 \pm 2.10^{\mathrm{A}-\mathrm{D} \text { ab }}$ & $72.50 \pm 2.40^{\mathrm{A}-\mathrm{D} \text { a }}$ & $71.25 \pm 1.44^{\mathrm{A}-\mathrm{D} \text { ab }}$ & $69.17 \pm 1.61^{\mathrm{C}-\mathrm{E} b}$ \\
\hline 7.5 & $55.00 \pm 1.54^{\mathrm{G}-\mathrm{I} \text { ab }}$ & $48.75 \pm 1.07 \mathrm{HI} \mathrm{c}$ & $69.38 \pm 2.15^{\mathrm{B}-\mathrm{D} \mathrm{ab}}$ & $71.88 \pm 2.68^{\mathrm{A}-\mathrm{D} \text { a }}$ & $68.13 \pm 1.53^{\mathrm{C}-\mathrm{F} \mathrm{ab}}$ & $67.50 \pm 1.61^{\mathrm{C}-\mathrm{F} b c}$ \\
\hline 10.0 & $45.63 \pm 1.11^{\mathrm{HI} \mathrm{c}}$ & $43.13 \pm 1.04^{\mathrm{Ic}}$ & $63.75 \pm 1.44^{\mathrm{D}-\mathrm{G} b}$ & $71.88 \pm 2.27^{\mathrm{A}-\mathrm{D} a}$ & $63.75 \pm 1.33^{\mathrm{D}-\mathrm{G} b}$ & $55.83 \pm 1.24^{\mathrm{F}-\mathrm{H} \mathrm{c}}$ \\
\hline
\end{tabular}

Data are reported as mean \pm standard deviation. Differences between different CCs or between different concentrations of the same CC were assessed for significance using two-way analysis of variance. Values with different lowercase letters (a-c) within a column or different uppercase letters (A-I) within a row differ significantly based on Fisher's least significant difference test at $p<0.05$. Liquid chromatography-tandem mass spectrometry detected 15 phenolic compounds from 6 different phenolic classes: phenolic aldehydes, bioflavonoids, flavonoid glycosides, flavonols, hydroxybenzoic acid and hydroxycinnamic acid. The phenolic composition and the content of these phenolic compounds ( $\mu \mathrm{g} / \mathrm{g}$ dry extract) at each CCs are given in Table 5.

Table 2. Allelopathic effect of different concentrations of aqueous extracts of cover crops on A. artemisiifolia shoot length, radicle length and seedling fresh weight.

\begin{tabular}{|c|c|c|c|c|c|c|c|}
\hline \multirow[b]{2}{*}{ Parameter } & \multirow[b]{2}{*}{$\begin{array}{c}\text { Concentration } \\
(w / v)\end{array}$} & \multicolumn{6}{|c|}{ Cover Crop (s) } \\
\hline & & $\begin{array}{l}\text { All Crops } \\
\text { Mixed Together } \\
\text { (CCMIX) }\end{array}$ & C. sativa & F. esculentum & G. abssynica & R. sativus & S. alba \\
\hline \multirow{7}{*}{$\begin{array}{l}\text { Shoot } \\
\text { length } \\
(\mathrm{cm})\end{array}$} & Control & $\underset{\mathrm{D}-\mathrm{N} \mathrm{a}-\mathrm{c}}{1.90} 0.52$ & & & & & \\
\hline & 0.5 & $2.34 \pm 0.17^{\mathrm{AB} a}$ & $1.54 \pm 0.29 \mathrm{Cd}$ & $1.92 \pm 0.67^{\mathrm{G}-\mathrm{K} a}$ & $2.09 \pm 0.86^{\mathrm{E}-\mathrm{H} \mathrm{a}}$ & $2.50 \pm 0.77 \mathrm{CD} \mathrm{a}$ & $\underset{\mathrm{L}-\mathrm{O} b \mathrm{bc}}{1.44} 0.35$ \\
\hline & 1.0 & $2.26 \pm 0.20 \mathrm{~A} \mathrm{a}$ & $1.36 \pm 0.24 \mathrm{Cd}$ & $1.81 \pm 0.70^{\mathrm{G}-\mathrm{L} a}$ & $2.05 \pm 0.62^{\mathrm{F}-\mathrm{I} a}$ & $2.36 \pm 0.74 \mathrm{C}-\mathrm{F} \mathrm{a}$ & $1.59 \pm 0.32^{\mathrm{K}-\mathrm{O} b}$ \\
\hline & 2.5 & $2.23 \pm 0.22 \mathrm{~B} \mathrm{a}$ & $1.39 \pm 0.11^{C d}$ & $1.68 \pm 0.54^{\mathrm{I}-\mathrm{M} \mathrm{a}}$ & $2.07 \pm 0.62^{\mathrm{F}-\mathrm{I} a}$ & $2.00 \pm 0.62^{\mathrm{F}-\mathrm{J} \mathrm{b}}$ & $1.53 \pm 0.35 \mathrm{~J}-\mathrm{N} \mathrm{b}$ \\
\hline & 5.0 & $2.08 \pm 0.22^{\mathrm{C}-\mathrm{E} \text { a }}$ & $1.10 \pm 0.15^{\mathrm{D}-\mathrm{G} \mathrm{d}}$ & $1.69 \pm 0.49 \mathrm{H}-\mathrm{M} \mathrm{a}$ & $1.99 \pm 0.68^{\mathrm{F}-\mathrm{J} \mathrm{a}}$ & $1.88 \underset{\mathrm{G}-\mathrm{K} b \mathrm{bc}}{ \pm} 0.34$ & $\underset{\mathrm{M}-\mathrm{P} \text { bc }}{ \pm} 0.27$ \\
\hline & 7.5 & $1.99 \pm 0.23^{\mathrm{F}-\mathrm{J} \mathrm{ab}}$ & $0.91 \pm 0.06^{\mathrm{P}-\mathrm{Se}}$ & $1.60 \pm 0.43^{\mathrm{J}-\mathrm{N} \text { a }}$ & $1.97 \pm 0.63^{\mathrm{F}-\mathrm{J} a}$ & $1.68 \pm 0.32^{\mathrm{I}-\mathrm{M} \mathrm{c}}$ & $1.15 \underset{\mathrm{O}-\mathrm{R}}{ \pm} \mathrm{cd}$ \\
\hline & 10.0 & $1.52 \pm 0.19^{\mathrm{M}-\mathrm{R} c}$ & $0.87 \pm 0.22^{\mathrm{Se}}$ & $1.64 \pm 0.50 \mathrm{~J}-\mathrm{N} \mathrm{a}$ & $1.85 \pm 0.48^{\mathrm{G}-\mathrm{K} a}$ & $1.27 \pm 0.22^{\mathrm{N}-\mathrm{R} \mathrm{d}}$ & $0.98 \pm 0.14^{\mathrm{R}-\mathrm{S} \mathrm{d}}$ \\
\hline \multirow{7}{*}{$\begin{array}{l}\text { Radicle } \\
\text { length } \\
(\mathrm{cm})\end{array}$} & Control & $\underset{\mathrm{D}-\mathrm{K} \text { a-c }}{3.79 \pm 0.42}$ & & & & & \multirow{3}{*}{$\begin{array}{c}5.46 \pm 1.09 \mathrm{~A} \mathrm{a} \\
4.73 \pm 0.50 \\
\mathrm{~A}-\mathrm{E} \mathrm{ab}\end{array}$} \\
\hline & 0.5 & $4.09 \pm 0.25^{\mathrm{L}-\mathrm{O} a}$ & $2.69 \pm 0.55^{\mathrm{N}-\mathrm{S} c}$ & $4.79 \pm 0.73^{\mathrm{A}-\mathrm{D} a}$ & $4.93 \pm 0.57^{\mathrm{A}-\mathrm{C} \mathrm{a}}$ & $4.41 \pm 0.92 \mathrm{AB} \mathrm{a}$ & \\
\hline & 1.0 & $4.45 \pm 0.46^{\mathrm{L}-\mathrm{O} c}$ & $2.76 \pm 0.28^{\mathrm{P}-\mathrm{T} \mathrm{c}}$ & $4.62 \pm 0.89^{\mathrm{B}-\mathrm{F} \mathrm{a}}$ & $4.63 \pm 0.61^{\mathrm{B}-\mathrm{F} a b}$ & $4.08 \pm 0.91^{\mathrm{A}-\mathrm{D} a}$ & \\
\hline & 2.5 & $\underset{\mathrm{L}-\mathrm{O} \text { cd }}{4.01} \underset{0.39}{ \pm}$ & $2.67 \underset{\mathrm{P}-\mathrm{T} \text { cd }}{ \pm} 0.26$ & $4.49 \pm 0.68^{\mathrm{C}-\mathrm{F} \mathrm{a}}$ & $\underset{\mathrm{A}-\mathrm{D} \mathrm{ab}}{4.79}=0.62$ & $4.05 \pm 0.59 \mathrm{~A}-\mathrm{D} \mathrm{a}$ & $\underset{\mathrm{A}-\mathrm{D} \mathrm{ab}}{4.83 \pm 0.72}$ \\
\hline & 5.0 & $2.48 \pm 0.18^{\mathrm{M}-\mathrm{Pd}}$ & $2.20 \pm 0.23^{\mathrm{ST} \mathrm{d}}$ & $\underset{\mathrm{D}-\mathrm{G} \text { ab }}{4.46} 0.86$ & $\underset{\mathrm{D}-\mathrm{H} \text { ab }}{4.32} 0.33$ & $3.65 \pm 0.37^{\mathrm{G}-\mathrm{Jb}}$ & $3.86 \pm 0.47^{\mathrm{F}-\mathrm{J} b c}$ \\
\hline & 7.5 & $2.00 \pm 0.40^{\mathrm{M}-\mathrm{R} \mathrm{d}}$ & $1.01 \pm 0.61^{\mathrm{Te}}$ & $3.28 \pm 0.58^{\mathrm{I}-\mathrm{K} b c}$ & $3.94 \pm 0.39^{\mathrm{E}-\mathrm{I} b c}$ & $2.35 \pm 0.17^{\mathrm{L}-\mathrm{N} \mathrm{c}}$ & $3.61 \pm 0.40^{\mathrm{H}-\mathrm{J} \mathrm{c}}$ \\
\hline & 10.0 & $1.32 \pm 0.35^{\mathrm{O}-\mathrm{T} \mathrm{e}}$ & $0.69 \pm 0.28^{\mathrm{Te}}$ & $2.27 \pm 0.40^{\mathrm{K}-\mathrm{Mc}}$ & $3.05 \pm 0.21 \mathrm{~J}-\mathrm{L} c$ & $1.23 \pm 0.29^{\mathrm{R}-\mathrm{T} \mathrm{d}}$ & $1.59 \pm 0.53^{\mathrm{N}-\mathrm{T} \mathrm{d}}$ \\
\hline \multirow{7}{*}{$\begin{array}{l}\text { Seedling } \\
\text { fresh } \\
\text { weight } \\
\text { (g) }\end{array}$} & Control & $\begin{array}{c}0.27 \pm \underset{\mathrm{A}-\mathrm{K} \mathrm{ab}}{ \pm 0.05} \\
0\end{array}$ & & & & & \multirow{6}{*}{$\begin{array}{c}0.34 \pm 0.04 \mathrm{~A}-\mathrm{C} \mathrm{a} \\
0.36 \pm 0.01 \mathrm{AB} \mathrm{a} \\
0.35 \pm 0.04 \mathrm{~A}-\mathrm{Ca} \\
0.33 \pm 0.06 \\
\mathrm{~A}-\mathrm{D} \mathrm{ab} \\
0.31 \pm 0.03 \\
\mathrm{~A}-\mathrm{F} \mathrm{ab}\end{array}$} \\
\hline & 0.5 & $0.40 \pm 0.17^{\mathrm{C}-\mathrm{I} a}$ & $0.31 \pm 0.04^{\mathrm{B}-\mathrm{G} \mathrm{a}}$ & $0.30 \pm 0.03^{\mathrm{B}-\mathrm{F} \mathrm{a}}$ & $0.33 \pm 0.07^{\mathrm{A}-\mathrm{D} a}$ & $0.32 \pm 0.04 \mathrm{~A}-\mathrm{E}$ a & \\
\hline & 1.0 & $0.49 \pm 0.11 \mathrm{~A} \mathrm{a}$ & $0.23 \pm 0.07^{\mathrm{E}-\mathrm{I} \text { a }}$ & $0.30 \pm 0.01^{\mathrm{B}-\mathrm{F} \mathrm{a}}$ & $0.35 \pm 0.07^{\mathrm{A}-\mathrm{Ca}}$ & $0.33 \pm 0.04^{\mathrm{A}-\mathrm{E} \text { a }}$ & \\
\hline & 2.5 & $0.43 \pm 0.13^{\mathrm{A}-\mathrm{C} a}$ & $0.22 \pm 0.07^{\mathrm{G}-\mathrm{K} a}$ & $0.31 \pm 0.06^{\mathrm{A}-\mathrm{F} \mathrm{a}}$ & $0.33 \pm 0.09^{\mathrm{A}-\mathrm{E} a}$ & $0.29 \pm 0.06^{\mathrm{B}-\mathrm{F} \mathrm{ab}}$ & \\
\hline & 5.0 & $0.30 \pm 0.16^{\mathrm{I}-\mathrm{K} \mathrm{b}}$ & $0.20 \pm 0.11^{\mathrm{G}-\mathrm{K} c}$ & $0.33 \pm 0.04^{\mathrm{A}-\mathrm{E} \text { a }}$ & $0.32 \pm 0.04^{\mathrm{A}-\mathrm{F} \mathrm{a}}$ & $0.29 \pm 0.04^{\mathrm{B}-\mathrm{F} \mathrm{ab}}$ & \\
\hline & 7.5 & $0.27 \pm 0.17^{\mathrm{KLb} c}$ & $0.17 \pm 0.01^{\mathrm{Mc}}$ & $0.32 \pm 0.05^{\mathrm{A}-\mathrm{D} \mathrm{a}}$ & $0.31 \pm 0.03^{\mathrm{A}-\mathrm{F} \mathrm{a}}$ & $0.23 \pm 0.01^{\mathrm{F}-\mathrm{J} b c}$ & \\
\hline & 10.0 & $0.22 \pm 0.15^{\mathrm{J}-\mathrm{L} b c}$ & $0.13 \pm 0.09 \mathrm{LMc}$ & $0.27 \pm 0.03^{\mathrm{B}-\mathrm{H} \mathrm{a}}$ & $0.28 \pm 0.03^{\mathrm{B}-\mathrm{G} a}$ & $0.18 \pm 0.02 \mathrm{H}-\mathrm{Kc}$ & $0.25 \pm 0.01^{\mathrm{D}-\mathrm{I} \mathrm{c}}$ \\
\hline
\end{tabular}

Data are reported as mean \pm standard deviation. Differences between different CCs or between different concentrations of the same CC were assessed for significance using two-way analysis of variance. Values with different lowercase letters (a-e) within a column or different uppercase letters (A-T) within a row differ significantly based on Fisher's least significant difference test at $p<0.05$. Liquid chromatography-tandem mass spectrometry detected 15 phenolic compounds from 6 different phenolic classes: phenolic aldehydes, bioflavonoids, flavonoid glycosides, flavonols, hydroxybenzoic acid and hydroxycinnamic acid. The phenolic composition and the content of these phenolic compounds ( $\mu \mathrm{g} / \mathrm{g}$ dry extract) at each CCs are given in Table 5. 


\subsection{Allelopathic Effects of Aqueous Extracts on A. artemisiifolia Germination Rate}

The extracts differentially affected the germination rate of $A$. artemisiifolia depending on the CCs and concentration. The effect of all CC aqueous extracts was significant for concentrations of $5 \%$. A significant interaction extract concentration $\times$ CCs was observed for initial germination (t10) and mean germination ( $\mathrm{t} 50)$ of A. artemisiifolia, so the values for each CCs and concentration are shown in Table 3. At concentrations of $0.5 \%-2.5 \%$, only C. sativa delayed the initial germination (Figure A1a), while C. sativa and CCMIX at these concentrations delayed mean germination, i.e., the number of days at which $50 \%$ of the seed sown has germinated (Figure A1a,b). At concentrations of $1 \%$ or $2.5 \%$, extracts and almost all Brassicaceae CC delayed the initial or mean germination (Figure A1a,c,d). Additionally, the non-Brassicaceae CCs F. esculentum and G. abssynica delayed the initial and mean germination at concentrations $\geq 5 \%$ (Figure A1e,f); at concentrations of $0.5 \%$ and $1 \%$, G. abssynica significantly delayed the mean germination. Taken together, these results indicate that all CCs inhibited germination rate, and C. sativa again exerted the strongest effects.

\subsection{Allelopathic Effects of Aqueous Extracts of Individual Parts of C. sativa on Germination and Early Growth}

Based on the assays of germination and germination rate, C. sativa was selected for an assay in which individual plant parts were assessed for their allelopathic effects on $A$. artemisiifolia. The tetrazolium test showed no differences in the percentage of viable seeds between any of the $C$. sativa plant organ aqueous extracts and the control. However, the aqueous extracts of all plant parts (root, stem, leaf, fruit and whole plant) significantly reduced germination, shoot length, radicle length, and seedling fresh weight in almost all cases compared to the control (Table 4). However, the strongest effects against $A$. artemisiifolia were observed with extracts of $C$. sativa fruits, which inhibited germination, shoot length, radicle length and seedling fresh weight by $75 \%, 57.3 \%, 90.9 \%$ and $75.7 \%$, respectively. These extracts showed an even stronger inhibitory effect on A. artemisiifolia than the extracts prepared from all parts of the C. sativa plant, which was particularly effective for final germination (75\% inhibition when treated with fruit extracts vs. $54 \%$ when treated with the extract of the whole plant.) Leaf and root aqueous extracts of $C$. sativa were similar in their effect on early growth inhibition of $A$. artemisiifolia, whereas the stem extract of $C$. sativa only significantly reduced the radical length and seedling fresh weight of $A$. artemisiifolia by respectively, $32.4 \%$ and $65.6 \%$. 
Table 3. The effect of aqueous extracts of CCs on the number of days needed for $10 \%\left(\mathrm{t}_{10}\right)$ or $50 \%\left(\mathrm{t}_{50}\right)$ of A. artemisiifolia seeds to germinate.

\begin{tabular}{|c|c|c|c|c|c|c|c|c|c|c|c|c|}
\hline \multirow{3}{*}{$\begin{array}{l}\text { Aqueous } \\
\text { Extract Con- } \\
\text { centration } \\
(w / v)\end{array}$} & \multicolumn{12}{|c|}{ Cover Crop (s) } \\
\hline & \multicolumn{2}{|c|}{$\begin{array}{l}\text { All Crops Mixed Together } \\
\text { (CCMIX) }\end{array}$} & \multicolumn{2}{|c|}{ C. sativa } & \multicolumn{2}{|c|}{ F. esculentum } & \multicolumn{2}{|c|}{ G. abssynica } & \multicolumn{2}{|c|}{ R. sativus } & \multicolumn{2}{|c|}{ S. alba } \\
\hline & $\mathbf{t}_{10}$ & $t_{50}$ & $t_{10}$ & $\mathbf{t}_{50}$ & $\mathbf{t}_{10}$ & $\mathbf{t}_{50}$ & $t_{10}$ & $t_{50}$ & $\mathbf{t}_{10}$ & $t_{50}$ & $t_{10}$ & $\mathbf{t}_{50}$ \\
\hline Control & $3.156^{\text {PR ab }}$ & $5.520^{\mathrm{Ta}}$ & $3.205^{\text {PR ab }}$ & $5.882^{\mathrm{P}-\mathrm{T} \text { a-c }}$ & $3.245^{\mathrm{PR} \mathrm{ab}}$ & $5.717^{\mathrm{R}-\mathrm{T} a b}$ & $3.067^{\mathrm{Ra}}$ & $5.575^{\mathrm{ST} \text { a }}$ & $3.152^{\mathrm{PR} a b}$ & $5.615^{\mathrm{ST} \mathrm{a}}$ & $3.237^{\mathrm{PR} a b}$ & $5.605^{\mathrm{ST} \mathrm{a}}$ \\
\hline 0.5 & $3.487^{\mathrm{O}-\mathrm{R} \mathrm{a}-\mathrm{c}}$ & $6.514^{\mathrm{I}-\mathrm{R} \mathrm{c}-\mathrm{g}}$ & $4.614^{\mathrm{E}-\mathrm{I} \text { h-l }}$ & $7.597^{\text {C-G k-p }}$ & $3.390^{\mathrm{O}-\mathrm{R} \mathrm{a}-\mathrm{c}}$ & $6.120^{\mathrm{M}-\mathrm{T} \text { a-e }}$ & $3.595^{\mathrm{N}-\mathrm{R} \mathrm{a}-\mathrm{d}}$ & $8.210^{\mathrm{K}-\mathrm{S}} \mathrm{o}-\mathrm{t}$ & $3.296^{\mathrm{PR} \mathrm{ab}}$ & $5.692^{\mathrm{R}-\mathrm{T}} \mathrm{ab}$ & $3.514^{\mathrm{O}-\mathrm{R} \text { a-c }}$ & $6.163^{\mathrm{L}-\mathrm{T}} \mathrm{a}-\mathrm{e}$ \\
\hline 1.0 & 3.729 L-R b-f & $6.828^{\mathrm{G}-\mathrm{N} \mathrm{e}-\mathrm{j}}$ & $4.596^{\mathrm{E}-\mathrm{I} h-1}$ & $7.437^{\mathrm{D}-\mathrm{H} \mathrm{i}-\mathrm{n}}$ & $3.515^{\mathrm{O}-\mathrm{R} \text { ab }}$ & $5.983^{\mathrm{O}-\mathrm{T} \mathrm{a}-\mathrm{d}}$ & $3.634^{\mathrm{M}-\mathrm{R} \text { a-e }}$ & $6.400^{\mathrm{K}-\mathrm{S} b-\mathrm{f}}$ & $3.785^{\mathrm{K}-\mathrm{P} \mathrm{b}-\mathrm{g}}$ & $6.165^{\mathrm{L}-\mathrm{T} \text { a-e }}$ & $4.003^{\mathrm{I}-\mathrm{O}-\mathrm{c}}$ & $6.380^{\mathrm{K}-\mathrm{Sb}-\mathrm{f}}$ \\
\hline 2.5 & $3.632 \mathrm{M}-\mathrm{R}$ a-e & $6.681^{\mathrm{D}-\mathrm{H} \mathrm{d}-\mathrm{h}}$ & $5.036^{\text {B-E 1-o }}$ & $8.177^{\text {A-D n-s }}$ & $3.484^{\mathrm{O}-\mathrm{R} \mathrm{a}-\mathrm{c}}$ & $6.058^{\mathrm{N}-\mathrm{T} \text { a-d }}$ & $3.522^{\mathrm{O}-\mathrm{R} \text { a-c }}$ & $5.928^{\mathrm{O}-\mathrm{T} \mathrm{a}-\mathrm{c}}$ & $4.410^{\mathrm{E}-\mathrm{K} g-1}$ & $6.946^{\text {F-M f-k }}$ & $3.579 \mathrm{~N}-\mathrm{R}$ a-d & $5.774^{\mathrm{R}-\mathrm{Ta}-\mathrm{C}}$ \\
\hline 5.0 & $4.522^{\mathrm{E}-\mathrm{I} h-1}$ & $7.787^{\mathrm{A}-\mathrm{E} \mathrm{m}-\mathrm{s}}$ & 5.607 AB op & $8.447 \mathrm{AB} \mathrm{r-t}$ & $4.286^{\mathrm{G}-\mathrm{M} \mathrm{e}-\mathrm{j}}$ & $7.171^{\mathrm{E}-\mathrm{K} \mathrm{g}-\mathrm{m}}$ & $4.223 \mathrm{H}-\mathrm{N} \mathrm{d}$-i & $6.954^{\mathrm{F}-\mathrm{L} \text { f-1 }}$ & $4.908^{C-G j-n}$ & $7.695^{\text {B-F 1-p }}$ & $4.211^{\mathrm{H}-\mathrm{N} \mathrm{d}-\mathrm{i}}$ & $7.076^{\mathrm{E}-\mathrm{K} \mathrm{f}-\mathrm{m}}$ \\
\hline 7.5 & $5.323^{\mathrm{A}-\mathrm{D} \mathrm{m}-\mathrm{p}}$ & $8.473 \mathrm{AB}$ st & $5.529^{\mathrm{A}-\mathrm{C} n-\mathrm{p}}$ & $8.591^{\mathrm{A} \mathrm{t}}$ & $4.824^{\mathrm{D}-\mathrm{H} i-\mathrm{m}}$ & $7.468^{\mathrm{C}-\mathrm{H} \mathrm{j-O}}$ & $4.037^{\mathrm{I}-\mathrm{O} c-\mathrm{h}}$ & $6.697 \mathrm{H}-\mathrm{P} \mathrm{d}-\mathrm{i}$ & $5.473^{\mathrm{A}-\mathrm{D} \mathrm{m}-\mathrm{p}}$ & $8.293^{\mathrm{A}-\mathrm{C} p-\mathrm{t}}$ & $4.237^{\mathrm{H}-\mathrm{N} \mathrm{d}-\mathrm{i}}$ & $6.727^{\mathrm{H}-\mathrm{O} \mathrm{d}-\mathrm{j}}$ \\
\hline
\end{tabular}

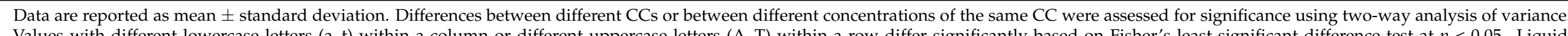

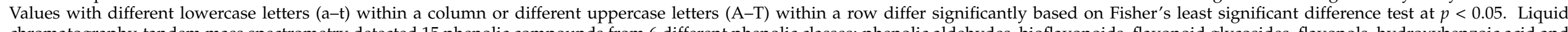
chromatography-tandem mass spectrometry detected 15 phenolic compounds from 6 different phenolic classes: phenolic aldehydes, bioflavonoids,

hydroxycinnamic acid. The phenolic composition and the content of these phenolic compounds ( $\mu \mathrm{g} / \mathrm{g}$ dry extract) at each CCs are given in Table 5. 
Table 4. Effect of aqueous extracts of individual parts of $C$. sativa at $0.1 \mathrm{~g} \mathrm{~mL}^{-1}$ on A. artemisiifolia weed radicle length, coleoptile length and whole fresh seedling weight.

\begin{tabular}{|c|c|c|c|c|c|c|}
\hline \multirow{2}{*}{ Parameters } & \multirow{2}{*}{ Control } & \multicolumn{5}{|c|}{ Camelina sativa Aqueous Extracts } \\
\hline & & Root & Stem & Leaf & Fruit & Whole Plant \\
\hline Viable seed (\%) & $95.2 \pm 5.22^{a}$ & $95.3 \pm 3.01^{a}$ & $93.3 \pm 3.27^{\mathrm{a}}$ & $95.2 \pm 6.57^{\mathrm{a}}$ & $92.8 \pm 5.22^{a}$ & $90.7 \pm 6.02^{a}$ \\
\hline Germinated seed (\%) & $73.6 \pm 6.69^{a}$ & $58.7 \pm 10.33^{b}$ & $63.3 \pm 5.89^{a, b}$ & $55.2 \pm 7.69^{b}$ & $18.4 \pm 8.76^{\mathrm{c}}$ & $54.0 \pm 10.04^{b}$ \\
\hline Shoot length $(\mathrm{cm})$ & $1.43 \pm 0.11^{\mathrm{a}}$ & $0.65 \pm 0.36^{b}$ & $1.25 \pm 0.57^{\mathrm{a}}$ & $0.66 \pm 0.39^{b}$ & $0.61 \pm 0.40^{b}$ & $0.52 \pm 0.34^{b}$ \\
\hline Radical length (cm) & $3.55 \pm 0.70^{\mathrm{a}}$ & $0.95 \pm 0.47^{b, c}$ & $1.22 \pm 0.69^{b}$ & $1.06 \pm 0.46^{\mathrm{b}}$ & $0.32 \pm 0.13^{\mathrm{d}}$ & $0.41 \pm 0.09^{c, d}$ \\
\hline Seedling fresh weight $(\mathrm{g})$ & $0.37 \pm 0.07^{\mathrm{a}}$ & $0.16 \pm 0.07^{c}$ & $0.25 \pm 0.07^{\mathrm{b}}$ & $0.14 \pm 0.07^{\mathrm{c}}$ & $0.09 \pm 0.04^{\mathrm{c}}$ & $0.12 \pm 0.05^{\mathrm{c}}$ \\
\hline
\end{tabular}

Data are reported as mean \pm standard deviation. The experiment was performed in 4 replicates of 25 seeds each and performed twice. Differences between different plant parts of $C$. sativa on germination and early growht of $A$. artemisiifolia were assessed for significance using one-way analysis of variance (ANOVA) completed with Fisher's least significant difference (LSD) test, $p<0.05$. Means in the same row marked by different letters $(\mathrm{a}-\mathrm{d})$ differ significantly $(p<0.05)$. Liquid chromatography-tandem mass spectrometry detected 13 phenolic compounds from 5 different phenolic classes in C. sativa dry extracts: phenolic aldehydes, flavonoid glycosides, flavonols, hydroxybenzoic acid and hydroxycinnamic acid. The phenolic composition and the content of these phenolic compounds ( $\mu \mathrm{g} / \mathrm{g}$ dry extract) are given in Table 5.

\subsection{Chemical Analyses of Aqueous CC Extracts and Correlation with Allelopathic Effects on A. artemisiifolia Radicle Length}

Extracts were found to contain 15 phenolic compounds falling into 6 chemical classes (Table 5): phenolic aldehydes, bioflavonoids, flavonoid glycosides, flavonols, hydroxybenzoic acids and hydroxycinnamic acids. Of the total of 15 phenolics detected, 13 were present in the C. sativa, S. alba and G. abssynica extracts; 12 in the F. esculentum and R. sativus extracts; and 11 in CCMIX. The CC extracts varied significantly in phenolic content, with the following CCs showing significantly more of the indicated phenolics than other CCs: S. alba, kaempferol $(46.5 \mu \mathrm{g} / \mathrm{g})$, gallic acid $(65.5 \mu \mathrm{g} / \mathrm{g})$, and p-hydroxybenzoic acid $(222.3 \mu \mathrm{g} / \mathrm{g}) ;$ F. esculentum, rutin $(1844.3 \mu \mathrm{g} / \mathrm{g})$, quercetin $8.96 \times 10^{5}\left(895.6 \times 10^{3} \mu \mathrm{g} / \mathrm{g}\right)$, quercetin $(135.8 \mu \mathrm{g} / \mathrm{g})$ and protocatechuic acid $(386.3 \mu \mathrm{g} / \mathrm{g}) ;$ R. sativa, p-coumaric acid $(84.5 \mu \mathrm{g} / \mathrm{g}) ;$ C. sativa, vanilin $(44.8 \mu \mathrm{g} / \mathrm{g})$, syringic acid $(27.3 \mu \mathrm{g} / \mathrm{g})$ and chlorogenic acid $(79.3 \mu \mathrm{g} / \mathrm{g})$; and CCMIX, caffeic acid $(134.8 \mu \mathrm{g} / \mathrm{g})$, sinapinic acid $(11.8 \mu \mathrm{g} / \mathrm{g})$ and ferulic acid $(1143 \mu \mathrm{g} / \mathrm{g})$.

The most abundant phenol in CC extracts was quercetin, whose concentration ranged from $6.8 \times 10^{4}-8.95 \times 10^{5}$. The content of most of the other detected phenols was less than $100 \mu \mathrm{g} / \mathrm{g}$. Sinapinic acid was detected in only two extracts: $S$. alba $(4.0 \mu \mathrm{g} / \mathrm{g})$ and CCMIX (11.8). 
Table 5. Quantification of phenolics in aqueous extracts from six cover crops.

\begin{tabular}{|c|c|c|c|c|c|c|c|}
\hline \multirow{2}{*}{ Chemical Class } & \multirow{2}{*}{ Compound } & \multicolumn{6}{|c|}{ Content of Phenolic Compounds ( $\mu \mathrm{g} / \mathrm{g}$ of Dry Extract) } \\
\hline & & S. alba & F. esculentum & R. sativa & G. abssynica & C. sativa & CCMIX \\
\hline Phenolic aldehydes & Vanillin & $19.0 \pm 0.28^{b}$ & $13.3 \pm 0.22^{c}$ & $19.0 \pm 0.23^{b}$ & $13.8 \pm 0.11^{\mathrm{c}}$ & $44.8 \pm 0.39^{\mathrm{a}}$ & n.d. ${ }^{\mathrm{d}}$ \\
\hline Bioflavonoids & Kaempferol & $46.5 \pm 0.59^{\text {a }}$ & n.d. ${ }^{c}$ & n.d. ${ }^{c}$ & $40.0 \pm 0.44^{\mathrm{b}}$ & n.d. ${ }^{c}$ & n.d. ${ }^{c}$ \\
\hline Flavonoid glycosides & Rutin & n.d. ${ }^{\mathrm{e}}$ & $1844.3 \pm 1.48^{\mathrm{a}}$ & $6.0 \pm 0.09^{d}$ & $28.8 \pm 0.25^{c}$ & $413.5 \pm 0.86^{\mathrm{b}}$ & $416.5 \pm 0.87^{b}$ \\
\hline \multirow{4}{*}{ Flavonols } & Quercetin & $71.6 \times 10^{3} \pm 21.18^{\mathrm{d}}$ & $895.6 \times 10^{3} \pm 10.90^{a}$ & $189.9 \times 10^{3} \pm 25.15^{b}$ & $980.5 \pm 35.65^{\mathrm{e}}$ & $68.8 \pm 0.75^{\mathrm{f}}$ & $88.8 \times 10^{3} \pm 5.25^{c}$ \\
\hline & Quercitin & $40.8 \pm 0.86^{c}$ & $135.8 \pm 1.11^{\mathrm{a}}$ & $8.3 \pm 0.05^{\mathrm{e}}$ & $7.0 \pm 0.09^{\mathrm{e}}$ & $33.3 \pm 0.48^{\mathrm{d}}$ & $59.5 \pm 0.60^{\mathrm{b}}$ \\
\hline & Gallic acid & $65.5 \pm 0.47^{\mathrm{a}}$ & $32.3 \pm 0.65^{b}$ & $18.5 \pm 0.43^{c}$ & $17.5 \pm 0.34^{c}$ & $17.5 \pm 0.35^{c}$ & $26.3 \pm 0.50^{b}$ \\
\hline & Protocatechuic acid & $55.5 \pm 0.60^{\mathrm{e}}$ & $386.3 \pm 0.74^{\mathrm{a}}$ & $63.5 \pm 0.76^{\mathrm{d}}$ & $38.3 \pm 0.53^{f}$ & $100.5 \pm 0.50^{c}$ & $113.5 \pm 0.52^{b}$ \\
\hline \multirow{4}{*}{ Hydroxybenzoic acids } & p-Hydroxybenzoic acid & $222.3 \pm 0.86^{a}$ & $38.5 \pm 0.38^{c}$ & $154.3 \pm 1.0^{\mathrm{b}}$ & $35.8 \pm 0.43^{c}$ & $36.8 \pm 0.45^{c}$ & $27.0 \pm 0.43^{\mathrm{d}}$ \\
\hline & p-coumaric acid & $25.3 \pm 0.53^{c}$ & $26.5 \pm 0.43^{c}$ & $84.5 \pm 0.59^{\mathrm{a}}$ & $16.3 \pm 0.24^{\mathrm{d}}$ & $74.8 \pm 0.61^{\mathrm{b}}$ & $29.5 \pm 0.30^{c}$ \\
\hline & Chlorogenic acid & n.d. ${ }^{d}$ & $87.8 \pm 0.47^{c}$ & n.d. ${ }^{d}$ & $37.5 \pm 0.33^{c}$ & $1057.0 \pm 1.58^{a}$ & $587.8 \pm 1.20^{\mathrm{b}}$ \\
\hline & Vanillic acid & $31.8 \pm 0.56^{c}$ & n.d. d & $37.0 \pm 0.22 \mathrm{~b}$ & n.d. d & $79.3 \pm 0.65^{\mathrm{a}}$ & n.d. d \\
\hline \multirow[t]{3}{*}{ Hydroxycinnamic acids } & Caffeic acid & $14.5 \pm 0.11^{\mathrm{f}}$ & $46.3 \pm 0.38^{c}$ & $26.8 \pm 0.30^{\mathrm{e}}$ & $35.0 \pm 0.52 \mathrm{~d}$ & $102.5 \pm 0.65^{b}$ & $134.8 \pm 0.60^{\mathrm{a}}$ \\
\hline & Sinapinic acid & $4.0 \pm 0.09^{b}$ & n.d. ${ }^{c}$ & n.d. ${ }^{c}$ & n.d. c ${ }^{c}$ & n.d. ${ }^{c}$ & $11.8 \pm 0.07^{\mathrm{a}}$ \\
\hline & Ferulic acid & $39.8 \pm 0.34^{c}$ & $11.8 \pm 0.19^{\mathrm{d}}$ & $552.0 \pm 1.11^{b}$ & $2.5 \pm 0.05^{\mathrm{e}}$ & $35.5 \pm 0.33^{c}$ & $1143.0 \pm 2.86^{\mathrm{a}}$ \\
\hline
\end{tabular}

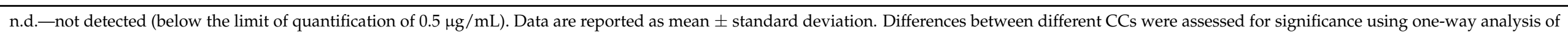
variance. Values with different lowercase letters (a-f) within a row differ significantly based on Fisher's least significant difference test at $p<0.05$. 


\section{Discussion}

The present study confirmed Brassicaceae CCs as allelopathic plants $[19,20]$ for suppressing the germination and early growth of A. artemisiifolia. C. sativa, S. alba and R. sativus inhibited early growth of $A$. artemisiifolia better than non-Brassicaceae CCs, which is also consistent with previous studies using other weeds as test species $[10,22]$. However, our study showed that the allelopathic potential of CCs strongly depends on their concentration in the aqueous extracts. With the exception of $C$. sativa, almost all CCs, and especially $F$. esculentum and G. abssynica, tested at lower concentrations slightly stimulated the early growth of A. artemisiifolia, which has also been explained previously by the fact that the same compound may be inhibitory at a high concentration, stimulatory at a low concentration or have no effect at other concentrations [23]. Among the Brassicaceae CCs tested, we found that $C$. sativa had the strongest inhibitory potential against the germination and early growth of $A$. artemisiifolia. Indeed, its aqueous extracts reduced $A$. artemisiifolia fresh seedling weight by up to $82 \%$, more than any of the other CCs in this study.

C. sativa has usually been analyzed by virtue of its abundant oil content [26] or its use in seed mixtures for wildflower strips [27]. In our study, however, we discovered that it inhibited A. artemisiifolia germination and early growth more than any other CCs. As far as we know, this is the first report of $C$. sativa allelopathic effects against $A$. artemisiifolia and the most detailed comparison yet of its allelopathic potential with that of other Brassicaceae CCs. In previous work, aqueous extracts of C. sativa roots and shoots inhibited Avena fatua germination more strongly than Brassica napus did [28]. Another study showed that C. sativa strongly inhibited the grass weed species Echinochloa crus-galli L. and Setaria viridis L., and these effects were associated with phenols in C. sativa's leaves and reproductive organs [22]. The present study also showed the greatest reduction in germination, shoot and root length and seedling fresh weight when $A$. artemisiifolia seeds were treated with aqueous extracts of $C$. sativa fruits, suggesting that flowers are the plant organs richest in allelochemicals. This may suggest that if $C$. sativa is to be incorporated as a cover crop, the best time to do so is the flowering stage [22].

The allelopathic activity of Brassicaceae CCs is mediated by glucosinolates as well as by phenolic compounds in plant tissues; these phenolics suppress the synthesis of proteins and nucleic acids, and they inactivate several enzymes in growing plants [29-31]. Liquid chromatography-tandem mass spectrometry in the present study indicated the presence of 15 phenols that are candidate mediators of the allelopathy against $A$. artemisiifolia (Table 3). The results of the current study do not allow us to specifically conclude which phenolics most caused growth inhibition of $A$. artemisiifolia, but the phenolics detected here are well-known weed inhibitory agents. For example, vanilic acid damaged dry mass or chlorophyll a content of Echinochloa crus-galli or Galinsoga parviflora to a greater extent than other phenolics did [32]. Syringic acid, for its part, has been found to significantly reduce the seedling growth of Corchorus olitorius [33]. Caffeic, syringic and p-coumaric acids appear to help mediate the ability of B. nigra to inhibit various weed species [34], and our data suggest that the levels of these compounds in C. sativa are more than twice their levels in B. nigra. Kaempherol and gallic acid, which were most abundant in our S. alba extracts, have also shown allelopathic effects $[32,35,36]$. Thus, we attribute the sensitivity of early A. artemisiifolia growth to $S$. alba aqueous extracts to the presence of kaempherol and gallic acid and the sensitivity to $R$. sativus extracts to an abundance of p-coumaric acid.

High concentrations of aqueous CC extracts have been shown to cause embryo death in seeds [37], but we did not observe this. None of the tested Brassicaceae CCs completely inhibited A. artemisiifolia germination or seedling development. In fact, aqueous B. nigra extract at $0.04 \mathrm{~g} \mathrm{~mL}^{-1}$ completely inhibited the germination of T. alexandrinum, P. paradoxa and S.officinale seeds [34], whereas we did not observe that result when we used concentrations even 2.5-fold higher. Indeed, the tetrazolium test did not show differences in the percentage of viable seeds between untreated seeds and seeds treated with extracts from $C$. 
sativa root, stem leaf, fruit as well as from the whole plant (Table 4). A. artemisiifolia may be less sensitive to aqueous CC extracts, which is consistent with the idea that weed species may vary in their sensitivity to allelochemicals [25,38]. Differences in seed size might be a reason, with smaller seeds more susceptible to allelopathic compounds [39]. A. artemisiifolia has approximately 10-15-fold greater mass weight (ca. 4-5 g) than many weed species [40]. Further studies should examine whether any CCs can inhibit A. artemisiifolia more than the CCs tested here and whether the susceptibility to allelochemicals depends on seed weight, size, texture, color, degree of dormancy or shape [41]. It seems unlikely, however, that allelochemicals should totally inhibit weed growth [12].

CCs are usually more allelopathic to weed species when they are applied as a mixture than when they are used on their own $[10,14,34,42]$. However, this was not the case in the present study. Although CCMIX showed significantly higher content of caffeic, sinapininic and ferulic acids than any of the CCs on their own (Table 3), it did not exert the strongest allelopathic effects on A. artemisiifolia. Instead, C. sativa exerted stronger effects than CCMIX for all parameters measured (Table 2). Our laboratory results may not be entirely generalizable to the field since field studies suggest that CC mixtures consistently show greater stress tolerance than individual CCs [8]. Nevertheless, our results lead us to suggest increasing the seed proportion of $C$. sativa, the most allelopathic Brassicacea CC, beyond the $5 \%$ used in the Terra Gold CC seed mixture. It may even be quite effective to use $C$. sativa alone in fields highly infested with $A$. artemisiifolia, as in Croatia. Indeed, C. sativa requires less water than many $C C$ s and resists diseases and pests better [43], making it attractive in crop rotation.

Consistent with its excellent allelopathic potential, C. sativa delayed the seed germination of $A$. artemisiifolia even at the lowest concentration of $0.5 \%$ (Figure A1a). Delay in seed germination is crucial in weed control and can affect the ability of the seedlings to establish themselves in natural conditions [44]. Thus, under field conditions, C. sativa may emerge before $A$. artemisiifolia and develop a competitive advantage over it. This may help explain why the non-Brassicacee CCs F. esculentum and G. abssynica delayed germination of A. artemisiifolia (Figure A1e,f), which, therefore, are also attractive as CCs in the field, even if they showed relatively weak allelopathic effects in our laboratory experiments.

\section{Materials and Methods}

\subsection{Plant Material (Sources)}

The commercial CC product Tera Gold 14 (Terra Gold TG-14, Feldsaaten Freudenberger, Krefeld, Germany) was used. It is composed of 40\% Sinapis alba, 30\% Raphanus satious var. oleiformis, 20\% Fagopyrum esculentum, 5\% Camellina sativa and 5\% Guizotia abyssinica. The mixture was sown at $25 \mathrm{~kg} \mathrm{ha}^{-1}$ to a depth of $2-3 \mathrm{~cm}$ after wheat harvest at the Šašinovec Experimental Station ( $\left.45^{\circ} 51^{\prime} 05.2^{\prime \prime} \mathrm{N} 16^{\circ} 10^{\prime} 34.1^{\prime \prime} \mathrm{E}\right)$ at the University of Zagreb, Faculty of Agriculture, in August 2018. Soil type was silty clay loam (USDA, 1987), with pH $7.74\left(\mathrm{H}_{2} \mathrm{O}\right)$ and $7.04(\mathrm{KCl})$. Aerial parts of the CC mix were harvested separately in October 2018 at the growth stage BBCH 34-87. All CC plants from a $0.5 \mathrm{~m} \times 0.5 \mathrm{~m}$ square were collected at five locations. Collected materials were dried at $70{ }^{\circ} \mathrm{C}$ in an oven (UF 260, Memmert, Germany) until a constant weight was reached. Dry plant materials were chopped in a blender (GM 300 Grindomix Knife Mill, Retsch), ground in the blender and passed through a $0.5 \mathrm{~mm}$ sieve (SM 200, Retsch).

Mature, ripe seeds (achenes) of $A$. artemisiifolia L. were hand-collected from single populations of plants at the Šašinovec Experimental Station in October 2016. After collection, seeds were cleaned and stored in paper bags in darkness at room temperature until the start of the experiment. Seeds of $A$. artemisiifolia of uniform size and color, without visible signs of insect predation, were selected under a stereomicroscope. A. artemisiifolia germination was tested and found to be satisfactory for further seed bioassays. 


\subsection{Seed Bioassay}

The ground plant material was soaked in the dark at room temperature $\left(20 \pm 1{ }^{\circ} \mathrm{C}\right)$ and kept in distilled water for $24 \mathrm{~h}$ in a 1:10 ratio $\left(0.1 \mathrm{~g} \mathrm{~mL}^{-1}\right)$. The extracts were filtered through two-layer filter paper $\left(80 \mathrm{~g} / \mathrm{m}^{2}, 21 / \mathrm{N}\right.$, Munktell) to remove plant debris. The supernatant was stored in the dark at $4{ }^{\circ} \mathrm{C}$ until use. Pilot germination tests with aqueous extracts $\left(0.1 \mathrm{~g} \mathrm{~mL}^{-1}\right)$ of CCs, individually or all mixed together, inhibited A. artemisiifolia germination, root or shoot development (Table A1).

Seed bioassays were performed by mixing different amounts of each chosen plant powder in distilled water to concentrations $(w / v)$ of $0.5 \%, 1 \%, 2.5 \%, 5 \%, 7.5 \%$ or $10 \%$. A plant whose extracts showed the greatest inhibitory effect were further assessed by examining the effects of the aqueous extracts of root, stem, leaf or flower separately. In this plant part bioassay, the tetrazolium test [45] was performed to determine the percentage of viable seeds, i.e., to separate dead from dormant seeds.

Control Petri dishes contained distilled water. A. artemisiifolia seeds were soaked in a $2 \% \mathrm{KNO}_{3}$ solution for $24 \mathrm{~h}$ to alleviate their dormancy. A total of 40 soaked imbibed seeds were placed into each Petri dish ( $90 \mathrm{~mm}$ diameter), to which was added $5 \mathrm{~mL}$ of each plant seed solution. Dishes were kept in the climate chamber (HCP 108, Memmert, Germany) under the following conditions: photoperiod, $12 \mathrm{~h} / 12 \mathrm{~h}$; day temperature, $25^{\circ} \mathrm{C}$; night temperature, $15^{\circ} \mathrm{C}$; humidity, $70 \%$; and light intensity, $40-50 \mu \mathrm{mol} / \mathrm{m}^{2}$. All dishes were hermetically sealed with parafilm to avoid evaporation. Germinated seeds were counted every day over a two-week period. On the last day, the percentage of germination and early seedling growth were measured; the latter was measured in terms of shoot length, radicle length and seedling fresh weight. Germination rates were assessed by counting the number of germinated seeds at $24 \mathrm{~h}$ intervals during 14 days. Seeds were classified as germinated if they developed radicles $>1 \mathrm{~mm}$. The experimental design was a complete randomized block with four replications.

\subsection{Chemical Analyses of Aqueous Extracts}

\subsubsection{Standards and Reagents}

Reference standards of phenolic compounds were obtained from Sigma-Aldrich (Steinheim, Germany), Fluka Chemie (Buchs, Switzerland) or Chromadex (Santa Ana, CA, USA). HPLC-grade methanol was purchased from J.T. Baker (Deventer, The Netherlands), and p.a. formic acid and DMSO were obtained from Merck (Darmstadt, Germany).

\subsubsection{Liquid Chromatography-Tandem Mass Spectrometry}

Water extracts were filtered through $0.45-\mu \mathrm{m}$ filters of nylon with a diameter of 25 mm (KX Syringe Filter, catalogue no.ESF-NY-25-045-D, Kinesis, www.kinesis-group.com (accessed on 17 April 2021)). Fifteen working standards ranging from 0.001 to $10 \mu \mathrm{g} / \mathrm{mL}$ were prepared by serial 1:1 dilution of standard mixture with a 1:1 mixture of mobile phase A ( $0.1 \%$ aqueous formic acid) and B ( $0.1 \%$ formic acid in methanol). Samples and standards were analyzed using a 1260 Series high-performance liquid chromatography system (Agilent Technologies, Santa Clara, USA) coupled with a 6460A Triple Quad tandem mass spectrometer with an electrospray ion source (Agilent Technologies). This coupled system was controlled using MassHunter software (version B.06.00, Agilent Technologies). Samples $(5 \mu \mathrm{L})$ were injected into the system, and compounds were separated on a Zorbax Eclipse XDB-C18 column $(50 \times 4.6 \mathrm{~mm}, 1.8 \mu \mathrm{m})$ at $40{ }^{\circ} \mathrm{C}$. Mobile phase flow rate was $0.4 \mathrm{~mL} / \mathrm{min}$, and the following gradient was applied: at $0 \mathrm{~min}, 5 \% \mathrm{~B} ; 2 \mathrm{~min}, 5 \% \mathrm{~B} ; 10 \mathrm{~min}$, $50 \% \mathrm{~B} ; 12 \mathrm{~min}, 70 \% \mathrm{~B} ; 20 \mathrm{~min}, 90 \% \mathrm{~B}$; and re-equilibration time, $5 \mathrm{~min}$. Eluted components were detected using mass spectrometry with the following parameters: nebulization gas $\left(\mathrm{N}_{2}\right)$ pressure, $40 \mathrm{psi}$; drying gas $\left(\mathrm{N}_{2}\right)$ flow, $11 \mathrm{~L} / \mathrm{min}$; temperature, $350{ }^{\circ} \mathrm{C}$; and ion polarity, negative (ESI-). Data for compound detection, used in the dynamic MRM mode (retention time, precursor ion, product ion and Fragment and Collision energies), are given in Table 2. The ion polarity of all compounds was negative. Concentrations of all compounds were 
determined using the Agilent MassHunter Workstation Software-Quantitative Analysis (ver. B.06.00).

\subsection{Statistical Analysis}

The chromatography-mass spectrometry experiments were performed in triplicate. Data were expressed as means \pm standard deviation. Cumulative germination on a daily basis was analyzed using a logistic function in the Bioassay 97 statistical program (Onofri 2001), and the resulting germination time course was used to determine the time necessary for the germination of $10 \%\left(t_{10}\right)$ or $50 \%\left(t_{50}\right)$ of sown seeds. The normality and homogeneity of variance of all data were checked using the Kolmogorov-Smirnov and Levene tests. Differences in germination percentage, seedling growth, seedling fresh weight and cumulative germination between different CC species and concentrations were assessed for significance using two-way ANOVA using StatSoft, Inc. (2007) STATISTICA data analysis software system, www.statsoft.com (accessed on 17 April 2021), Tulsa OK, USA. Differences between treatments were also assessed for significance using Fisher's least significant difference test. For all statistical tests, differences associated with $p<0.05$ were considered significant.

\section{Conclusions}

A. artemisiifolia is a highly allergenic species around the world and the most abundant weed species among all summer crops in Croatia. Thus, the present study may help guide future work to examine how the allelopathic effects of CCs vary with abiotic and biotic factors that can degrade allelochemicals in the field. When CCs (C. sativa) are incorporated into the soil to provide organic matter and nutrients, our work suggests, at the flowering stage (Table 4). Such work may also guide the effective use of $C$. sativa applied directly as bioherbicides, but further studies are needed to specify which type of phenolic compounds is responsible for growth inhibition of $A$. artemisiifolia. This would be particularly helpful in areas with $A$. artemisiifolia-resistant biotypes [7]. Of course, CCs should be tested for unwanted allelopathy against arable crops [46]. Our preliminary results suggest that aqueous extracts of $C$. sativa do not affect germination or early growth of maize and soybean (data not shown). Consistently, previous work has shown that phenolic acids, even when applied directly at levels much higher than in soil, do not affect seedling growth or early plant growth of maize [47].

Author Contributions: Conceptualization, M.Š.; methodology, all authors; software, M.S.-K. and V.Š.; validation, M.S.-K., B.Š.T. and J.G.U.; formal analysis, all authors; investigation, E.B., L.R., J.L., V.Šs, M.Š., M.S.-K. and B.Š.T.; resources, M.Š., M.S.-K. and V.Š.; data curation, all authors; writing-original draft preparation, M.Š., writing—review and editing, M.Š. and M.S.-K.; visualization, M.Š.; project administration, M.Š.; funding acquisition, M.Š. All authors have read and agreed to the published version of the manuscript.

Funding: This research was assisted with funding by the Foundation of the Croatian Academy of Sciences and Arts and the Ministry of Education, Science and Technological Development of the Republic of Serbia.

Institutional Review Board Statement: Not applicable.

Informed Consent Statement: Not applicable.

Conflicts of Interest: The authors declare no conflict of interest. 


\section{Appendix A}

Table A1. Optimized dynamic MRM parameters.

\begin{tabular}{cccccc}
\hline Compound & $\begin{array}{c}\text { Precursor } \\
\boldsymbol{m} / \boldsymbol{z}\end{array}$ & $\begin{array}{c}\text { Product } \\
\mathbf{m} / \boldsymbol{z}\end{array}$ & $\begin{array}{c}\mathbf{( F r}) \\
\mathbf{( V )}\end{array}$ & $\begin{array}{c}\mathbf{C E} \\
\mathbf{( V )}\end{array}$ & $\begin{array}{c}\mathbf{t}_{\mathbf{R}} \\
(\mathbf{m i n})\end{array}$ \\
\hline Gallic acid & 169.0 & 125.0 & 90 & 10 & 3.67 \\
Protocatechuic acid & 153.0 & 109.0 & 105 & 9 & 6.27 \\
Chlorogenic acid & 353.0 & 191.0 & 100 & 10 & 8.54 \\
Vanillic acid & 167.0 & 108.0 & 100 & 15 & 9.14 \\
Caffeic acid & 179.0 & 135.0 & 100 & 10 & 9.14 \\
Syringic acid & 197.0 & 182.0 & 90 & 7 & 9.71 \\
Vanilin & 151.1 & 136.0 & 100 & 17 & \\
p-coumaric acid & 151.1 & 90.0 & 100 & 21 & 10.02 \\
Sinapinic acid & 163.0 & 119.0 & 90 & 9 & 10.68 \\
Ferulic acid & 223.0 & 193.0 & 100 & 17 & 11.13 \\
Rutin & 193.0 & 134.0 & 90 & 11 & 11.14 \\
Quercetin & 609.0 & 300.0 & 135 & 42 & 11.96 \\
Quercitin & 301.0 & 151.0 & 130 & 15 & 13.68 \\
Kaempferol & 447.0 & 300.0 & 190 & 27 & 12.07 \\
\hline & 285.0 & 285.0 & 130 & 0 & 14.25 \\
\hline
\end{tabular}

Table A2. The effects of different aqueous extracts on germination, shoot and radical length of A. artemisiifolia seeds.

\begin{tabular}{cccc}
\hline \multirow{2}{*}{ Cover Crop (s) } & \multicolumn{3}{c}{ Parameters } \\
\cline { 2 - 4 } & Germination (\%) & Shoot Length (cm) & Radical Length (cm) \\
\hline Control & $35.0 \pm 8.08^{\mathrm{a}}$ & $3.06 \pm 0.89^{\mathrm{a}}$ & $2.51 \pm 0.42^{\mathrm{a}}$ \\
CCmix & $23.0 \pm 3.06^{\mathrm{b}}$ & $0.90 \pm 0.05^{\mathrm{c}}$ & $1.05 \pm 0.11^{\mathrm{c}}$ \\
S. alba & $8.0 \pm 1.90^{\mathrm{c}}$ & $0.63 \pm 0.04^{\mathrm{c}, \mathrm{d}}$ & $0.10 \pm 0.00^{\mathrm{d}}$ \\
R. sativus & $1.0 \pm 0.06^{\mathrm{d}}$ & $0.00 \pm 0.00^{\mathrm{e}}$ & $0.27 \pm 0.08^{\mathrm{d}}$ \\
F. esculentum & $12.0 \pm 1.32^{\mathrm{c}}$ & $2.29 \pm 0.09^{\mathrm{b}}$ & $1.98 \pm 0.01^{\mathrm{b}}$ \\
C. sativa & $9.0 \pm 0.50^{\mathrm{c}}$ & $0.31 \pm 0.04^{\mathrm{d}}$ & $0.08 \pm 0.03^{\mathrm{d}}$ \\
G. abssynica & $9.0 \pm 1.72^{\mathrm{c}}$ & $0.92 \pm 0.08^{\mathrm{c}}$ & $0.82 \pm 0.04^{\mathrm{c}}$ \\
\hline
\end{tabular}

Data are reported as mean \pm standard deviation. Differences between different CCs were assessed for significance using one-way analysis of variance. Values with different lowercase letters (a-e) within a column differ significantly based on Fisher's least significant difference test at $p<0.05$. 


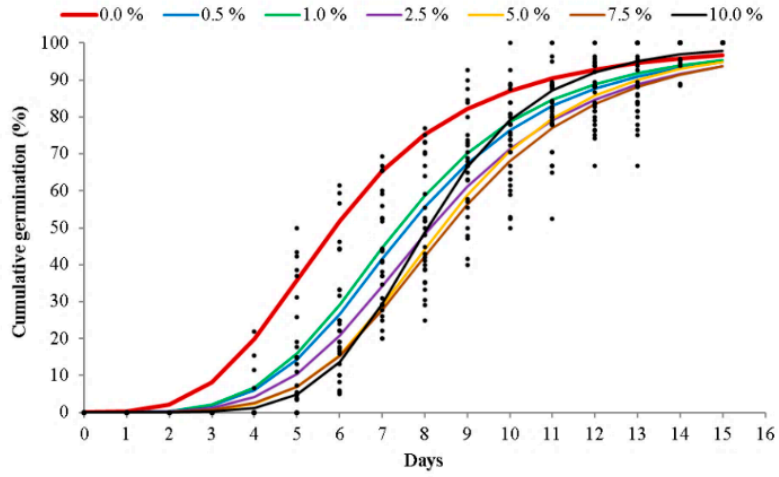

(a) Camelina sativa

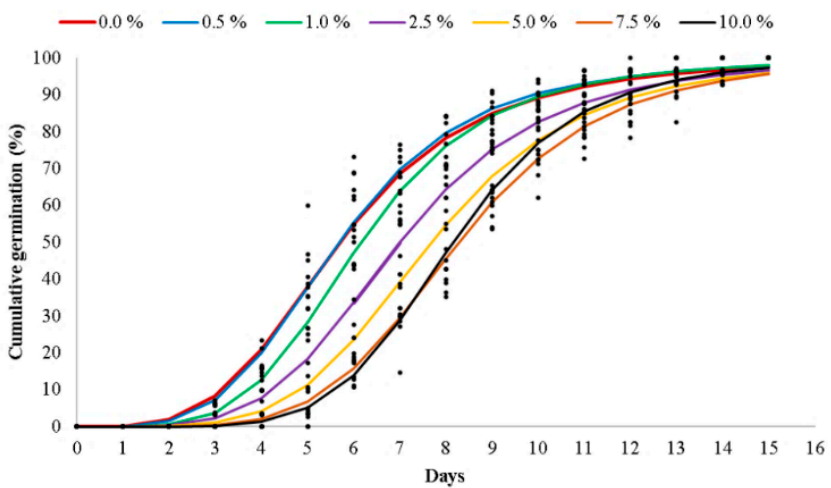

(c) Raphanus sativa

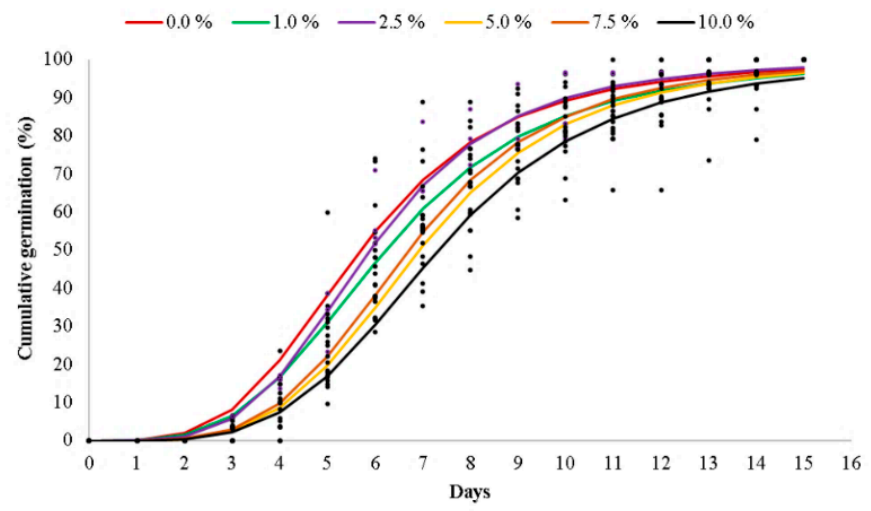

(e) Guizotia abssynica

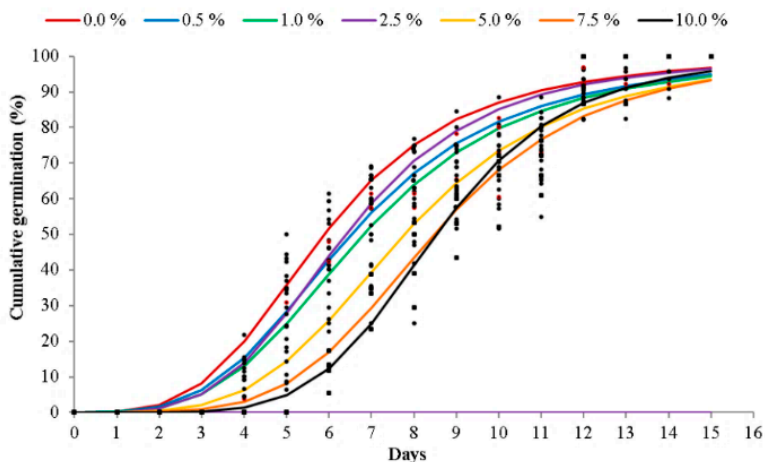

(b) Cover crop mixture

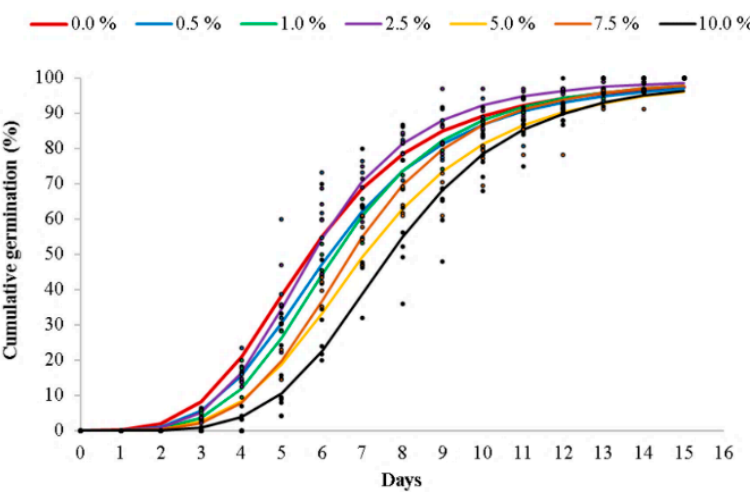

(d) Sinapis alba

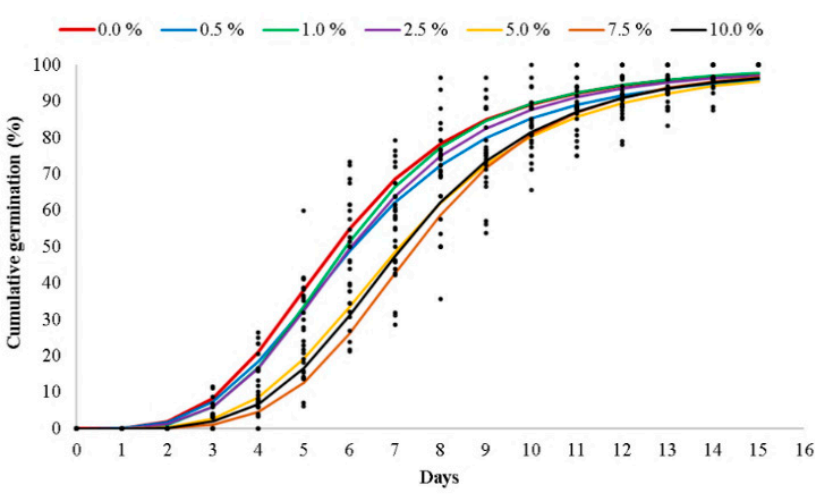

(f) Fagopyrum esculentum

Figure A1. The germination rate of $A$. artemisiifolia upon the aqueous extracts of CCs. The cumulative germination was analyzed using a logistic function in the Bioassay97 statistical program (Onofri 2001), obtaining germination time course under different concentrations of aqueous extracts. The solid line represents the fitted model, and the dots represent observed germination. Within each CC aqueous extract, the same line color the concentrations of aqueous extract $(0 \%-10 \%)$.

\section{References}

1. Essl, F.; Biró, K.; Brandes, D.; Broennimann, O.; Bullock, J.M.; Chapman, D.S.; Karrer, G. Biological flora of the British Isles: Ambrosia artemisiifolia. J. Ecol. 2015, 103, 1069-1098. [CrossRef]

2. Medzihradszky, Z.; Járai-Komlódi, M. I came from America-My name is Ambrosia-Some feature of the ragweed. In Proceedings of the 9th EWRS Symposium, Budapest, Hungary, 10-12 July 1995; pp. 57-63.

3. Šikoparija, B.; Radišić, P.; Pejak, T.; Šimić, S. Airborne grass and ragweed pollen in the southern Panonnian Valley-consideration of rural and urban environment. Ann. Agric. Environ. Med. 2006, 13, 263-266. [PubMed]

4. Lommen, S.T.E.; Caspar, A.H.; Jongejans, E.; Chauvel, B.; Leitsch-Vitalos, M.; Aleksanyan, A.; Scepanovic, M.; Onen, H.; Tokarska-Guzik, B.; Anastasiu, P.; et al. Explaining variability in the production of seed and allergenic pollen by invasive Ambrosia artemisiifolia across Europe. Biol. Invasions 2018, 5, 1475-1491. [CrossRef] 
5. Šarić, T.; Ostojić, Z.; Stefanović, L.; Deneva Milanova, S.; Kazinczi, G.; Tyšer, L. The changes of the composition of weed flora in Southeastern and Central Europe as affected by cropping practices. Herbologia 2011, 12, 8-12.

6. Galzina, N.; Barić, K.; Šćepanović, M.; Goršić, M.; Ostojić, Z. Distribution of Invasive Weed Ambrosia artemisiifolia L. in Croatia. Agric. Conspec. Sci. 2010, 75, 75-81.

7. Heap, I. The international Survey of Herbicide Resistance Weeds. 2021. Available online: www.weedscience.org (accessed on 17 January 2021).

8. Duke, S.O. Why have no new herbicide modes of action appeared in recent years? Pest Manag. Sci. 2012, 68, 505-512. [CrossRef] [PubMed]

9. Scavo, A.; Mauromicale, G. Integrated Weed Management in Herbaceous Field Crops. Agronomy 2020, 10, 466. [CrossRef]

10. Kunz, C.; Strum, D.; Varnholt, D.; Walker, F.; Gerhards, R. Allelopathic effects and weed suppressive ability of cover crops. Plant Soil Environ. 2016, 62, 60-66.

11. Triplett, G.B.; Warren, A.D. No-Tillage Crop Production: A Revolution in Agriculture! Agron. J. 2008. [CrossRef]

12. Scavo, A.; Restuccia, A.; Lombardo, S.; Fontanazza, S.; Abbate, C.; Pandino, G.; Anastasi, U.; Onofri, A.; Mauromicale, G. Improving soil health, weed management and nitrogen dynamics by Trifolium subterraneum cover cropping. Agron. Sustain. Dev. 2020, 40, 1-12. [CrossRef]

13. Finney, D.M.; Creamer, N.G. Weed Management on Organic Farms; The organic Production Publication Series; CEFS: Brussels, Belgium, 2008; pp. 1-34.

14. Macias, F.A.; Molinillo, M.G.; Oliveros-Bastidas, A.; Marin, D.; Chinchilla, D. Allelopathy. A natural strategy for weed control. Comm. Appl. Biol. Sci. 2004, 69, 13-23.

15. Jabran, K.; Mahajan, G.; Sardana, V.; Chauhan, B.S. Allelopathy for weed control in agricultural systems. Crop. Prot. 2015, 72, 57-65. [CrossRef]

16. Farooq, M.; Jabran, K.; Cheema, Z.A.; Wahid, A.; Siddique, K.H.M. The role of allelopathy in agricultural pest management. Pest Manag. Sci. 2011, 67, 493-506. [CrossRef] [PubMed]

17. Nichols, V.; Verhulst Cox, R.; Govaers, B. Weed dynamics in conservation agriculture principles: A review. Field Crop Res. 2015, 183, 56-68. [CrossRef]

18. Gentili, R.; Gilardelli, F.; Ciappetta, S.; Ghiani, A.; Citterio, S. Inducing competition: Intensive grassland seeding to control Ambrosia artemisiifolia. Weed Res. 2015, 55, 278-288. [CrossRef]

19. Hiridate, S. Isolation strategies for finding bioacitve compounds: Specific activirty vs. total activity. Chem. Soc. Symp. Ser. 2006, 927, 113-126.

20. Sherif, E.A.; Hegazy, A.K.; Gomaa, N.H.; Hassan, M.O. Allelopathic effect of Black Mustard Tissues and Root Exudates on some crops and weeds. Planta Daninha Vixoa MG 2013, 31, 11-19. [CrossRef]

21. Dhima, K.V.I.; Vasilakoglou, T.D.; Gatsis, E. Panou-Philotheou, Eleftherohorinos, I. Effects of aromatic plants incorporated as green manure on weed and maize development. Field Crops Res. 2009, 110, 235-241. [CrossRef]

22. Brijačak, E.; Košćak, L.; Šoštarčić, V.; Kljak, K.; Šćepanović, M. Sensitivity of yellow foxtail (Setaria glauca L) and barnyardgrass (Echninochloa crus-galli L.) to aqueous extracts or dry biomass of cover crop. J. Sci. Food Agric. 2020. [CrossRef]

23. Scavo, A.; Restuccia, A.; Pandino, G.; Onofri, A.; Mauromicale, G. Allelopathic effect of Cynara cardunculus L. leaf aqueous extracts on seed germination of some Medirettanean weed species. Ital. J. Agron. 2018, 3, 119-125.

24. Tessio, F.; Ferrero, A. Allelopathy, a chance for sustainable weed management. Int. J. Sust. Dev. World 2010, 17, 377-389. [CrossRef]

25. Gomaa, N.H.; Hassan, M.O.; Fahmy, G.M.; Gonzalez, L.; Hammouda, O.; Atteya, A.M. Allelopathic effect of Sonchus oleraceus L. on the germination and seedling growth of crop and weed species. Acta Bot. Bras. 2014, 25, 408-416. [CrossRef]

26. Francis, A.; Warwick, S.I. The biology of Canadian weeds. 142. Camelina alyssum (Mill.) Thell.; C. microcarpa Andrz. Ex DC.; C. sativa (L.) Crantz. Can. J. Plant Sci. 2009, 89, 791-810.

27. Kollmann, J.; Bassin, S. Effects of management on seed predation in wildflowerstrips in northern Switzerland. Agric. Ecocsyst. Environ. 2001, 83, 285-296. [CrossRef]

28. Walsh, K.D.; Sanderson, D.; Hall, L.M.; Mugo, S.; Hills, M.J. Allelopathic effects of camelina (Camelina sativa) and canola (Brassica napus) on wild oat, flax and radish. Allelopath. J. 2014, 33, 83-96.

29. Hiag, T. Allelochemicals in plants. In Allelopathy in Sustainable Agriculture and Forestry; Zeng, R.S., Mallik, A.U., Luo, S.S., Eds.; Springer: New York, NY, USA, 2008; pp. 63-104.

30. Quasem, J.G.; Foy, C.L. Weed allelopathy, its ecological impacts and future prospects: A review. Weed Biol. Manag. 2001, 4, 43-199. [CrossRef]

31. Chou, C.H. Introduction of allelopathy. In Allelopathy: A Physiological Process with Ecological Implications; Reigosa, M.J., Pedrol, N., Gonzales, L., Eds.; Springer: Dordrecht, The Netherlands, 2006; pp. 1-9.

32. Stupnicka-Rodzynkiewicz, E.; Dabkowska, T.; Stoklosa, A. The effect of selected phenolic compounds on the initial growth of four weed species. J. Plant Dis. Prot. 2006, 20, 479-486.

33. Fahmy, G.M.; Al-Sawaf, N.A.; Turki, H.; Ali, H.I. Allelopathic potential of Pluchea dioscordis (L.) DC. J. Appl. Sci. Res. 2012, 8 , 3129-3142.

34. Li, Z.H.; Wang, Q.; Ruan, X.; De Pan, C.; Jiang, D.-A. Phenolics and Plant Allelopathy. Molecules 2010, 15, 8933-8952. [CrossRef]

35. Turk, M.A.; Shatnawi, M.K.; Tawaha, A.M. Inhibitory effects of aqueous extracts of black mustard on germination and growth of alfalfa. Weed Biol. Manag. 2003, 3, 37-40. [CrossRef] 
36. Iqbal, Z.; Hiradate, S.; Noda, A.; Isojima, S.-I.; Fujii, Y. Allelopathic activity of buckwheat: Isolation and characterization of phenolics. Weed Sci. 2003, 51, 657-662. [CrossRef]

37. Einhellig, F.A. Mode of allelochemical action of phenolic compounds. In Allelopathy: Chemistry and Mode of Action of Allelochemicals; Macias, F.A., Galindo, J.C.G., Molinillo Jose, M.G., Eds.; CRC Press, LCC: London, UK, 2004.

38. Batish, D.R.; Kaur, S.; Singh, H.P.; Kohli, R.K. Nature of interference potential of leaf debris of Ageratum conyzoides. Plant Growth Regul. 2009, 57, 137-144. [CrossRef]

39. Liebman, M.; Davis, A. Integration of soil, crop and weed management in low-external-input farming systems. Weed Res. 2000, 40, 27-47. [CrossRef]

40. Šoštarčić, V.; Masin, R.; Turčinov, M.; Carin, N.; Šćepanović, M. Morfološka i funkcionalna intrapopulacijska varijabilnost sjemena korovne vrste Ambrosia artemisiifolia L. J. Cent. Eur. Agric. 2020, 21, 366-378. [CrossRef]

41. Haramoto, E.R.; Gallandt, E.R. Brassica cover cropping: I. Effect on weed and crop establishment. Weed Sci. 2005, 53, 695-701. [CrossRef]

42. Muscolo, A.; Panuccio, M.; Sidari, M. The effect of phenols on respiratory enzymes in seed germination. Plant Growth Regul. 2001, 35, 31-35. [CrossRef]

43. Sobiech, L.; Grzanka, M.; Popowska, D.K.; Rdzikowska, D.; Sobiech, Ł.; Grzanka, M.; Kurasiak-Popowska, D.; Radzikowska, D. Phytotoxic Effect of Herbicides on Various Camelina [Camelina sativa (L.) Crantz] Genotypes and Plant Chlorophyll Fluorescence. Agriculture 2020, 10, 185. [CrossRef]

44. Chaves, N.; Sosa, T.; Escudero, J.C. Plant growth inhibiting flavonoids in exudate of Cistus ladanifer and in associatedsoils. J. Chem. Ecol. 2001, 27, 623-631. [CrossRef]

45. ISTA. International Rules for Seed Testing; International Association for Seed Testing: Zurich, Switzerland, 2008.

46. Einhellig, F.A.; Rasmussen, J.A. Effects of three phenolic acids on chlorophyll content and growth of soybean and grain sorghum seedlings. J. Chem. Ecol. 1979, 5, 815-824. [CrossRef]

47. Krogmeir, M.J.; Bremner, J.M. Effects of phenolic acids on seed germination and seedling growth in soil. Biol. Fertil. Soils 1989, 8, 116-122. [CrossRef] 